\title{
Liouville theorems for harmonic maps
}

\author{
Zhiren $\operatorname{Jin}^{\star, \star \star}$ \\ Institute for Advanced Study, Princeton, NJ 08540, USA
}

Oblatum 28-XII-1990 \& 11-II-1991

Summary. We prove several Liouville theorems for harmonic maps between certain classes of Riemannian manifolds. In particular, the results can be applied to harmonic maps from the Euclidean space $\left(R^{m}, g_{0}\right)$ to a large class of Riemannian manifolds. Our assumptions on the harmonic maps concern the asymptotic behavior of the maps at $\infty$.

\section{Introduction}

In this paper, we investigate Liouville type properties for harmonic maps. That is, we study conditions for which a harmonic map $u$ between two Riemannian manifolds $\left(M^{m}, g\right)$ and $\left(N^{n}, h\right)$ must be a constant map. Let $g_{0}$ be the Euclidean metric on $R^{m}, m \geqq 3$. We can state two special cases of our results as follows:

Theorem A. Let $u:\left(R^{m}, g_{0}\right) \rightarrow\left(N^{n}, h\right)$ be a $C^{2}$ harmonic map. If $u(x) \rightarrow p_{0} \in N^{n}$ as $|x| \rightarrow \infty$, then $u$ is a constant map.

Theorem B. Suppose the sectional curvature of $N^{n}$ is bounded from above. Then for any $p \in N^{n}$, there is an (nonempty) open neighbourhood $U_{p} \subset N^{n}$, such that the family of open sets $\left\{U_{p} \mid p \in N^{n}\right\}$ has following property:

If $u:\left(R^{m}, g_{0}\right) \rightarrow\left(N^{n}, h\right)$ is a $C^{2}$ harmonic map, and for some $p \in N^{n}, u(x) \in U_{p}$ as $|x| \rightarrow \infty$ (i.e. there is $R>0$, such that $u\left(R^{m} \backslash B(R)\right) \subset U_{p}$ ), then $u$ is a constant map.

Recently there has been much interest in the Liouville type theorems for harmonic maps. For a detailed survey and progress in this direction, see the works by Hildebrandt [4], Eells and Lemaire [2]. Here we would like to mention that for all known results, the conditions on the harmonic maps can be divided into two kinds. The first of these conditions concerns the finiteness of the energy of the map. In this direction, a result of Schoen and Yau [10] shows that a harmonic map with

\footnotetext{
* Supported by NSF grant DMS-8610730

$\star \star$ Current address: Department of Mathematics, University of Michigan, Ann Arbor, Michigan 48109 , USA
} 
finite energy, from a complete manifold with nonnegative Ricci curvature to a manifold with nonpositive sectional curvature, must be a constant map. Also a theorem due to Garber, Ruijsenaars, Seiler and Burns [3] says that each harmonic map $u: R^{m} \rightarrow S^{m}$ with finite energy must be a constant map $(m>2)$. This latter result has been generalized by Hildebrandt [4] and Sealey [11] to cover harmonic maps from $R^{m}$ with certain globally conformal flat metrics to an arbitrary Riemannian manifold. The second of these conditions concerns the smallness of the whole image of the domain manifold under the harmonic map. In this direction, there are various results due to Cheng [1], Hildebrandt, Jost, Widman, Kaul $[4,5,6,7]$, and Yau [12]. Roughly speaking, their results show that if the image $u\left(M^{m}\right)$ is "small", then the harmonic map is a constant map. The basic definition for "smallness" is that $u\left(M^{m}\right)$ is contained in a geodesic ball $B_{p}(r)$ which does not intersect with the cut locus of $p$. (Here $r<\frac{\pi}{2} \sqrt{C_{N}}$ where $C_{N}$ is an upper bound for the sectional curvature of $N^{n}$ (see [2, pp. 398])).

In the present paper we are interested in finding other kinds of conditions which will imply the Liouville theorems for harmonic maps. In our results, instead of assuming the global "smallness" of the image, we will only prescribe the asymptotic behavior at infinity for the harmonic maps. For the proof of Theorem A, the idea is to analyse the growth rate of the energy of the harmonic map on balls. On the one hand, the monotonicity formula for harmonic maps implies a lower bound for the growth rate of the energy. On the other hand the assumptions at infinity imply an upper bound for the growth rate. The two bounds will be contradictory unless the harmonic map is a constant map. In the proof of Theorem B, we need a stronger lower bound for the growth rate; a crucial ingredient in its derivation is a result due to Schoen and Uhlenbeck [9, Theorem 2.2].

When $m=2$, neither Theorem A, nor Theorem B is true because the conformal invariance of the energy. But for $m=1$, conclusions of Theorem A and Theorem B are obvious, because any harmonic map $u: R^{1} \rightarrow\left(N^{n}, h\right)$ is a geodesic with constant speed.

It is clear that Theorem B is stronger than Theorem A. However, we will prove Theorem A in a more general setting, i.e. for harmonic maps $u:\left(R^{m}, f g_{0}\right) \rightarrow\left(N^{n}, h\right)$ with some positive functions $f$ (see Theorem 1). We strongly believe that Theorem $\mathrm{B}$ is true for the same class of domain manifolds as in Theorem 1, but we are not able to prove such a result at present.

\section{Notations and results}

Let $\left(M^{m}, g\right),\left(N^{n}, h\right)$ be Riemannian manifolds. A map $u:\left(M^{m}, g\right) \rightarrow\left(N^{n}, h\right)$ is called harmonic, if $u$ is a critical point of the functional:

$$
E(u)=\int_{M^{m}} e(u) \mathrm{dvol}_{M}
$$

where $e(u)$ is the energy density. In local coordinates, $e(u)$ can be written as

$$
e(u)=g^{i j}(x) h_{\alpha \beta}(u) \frac{\partial u^{\alpha}}{\partial x_{i}} \frac{\partial u^{\beta}}{\partial x_{j}} .
$$


Here and throughout the paper, the summation convention is used. Unless otherwise specified, repeated Latin letters are summed from 1 to $m$, repeated Greek letters are summed from 1 to $n$. Also, constants which only depend on the manifolds but not the harmonic maps will be denoted by $C$ throughout the paper.

For $\left(R^{m}, g_{0}\right)$ with the Euclidean metric $g_{0}, B(R)$ will be the ball centered at origin with radius $R$. In this paper we only consider the case that $M^{m}=R^{m}$ and $g=f g_{0}$ for some positive function $f$ defined on $R^{m}$. Then the energy density is

$$
e(u) \mathrm{dvol}_{M^{m}}=f^{\frac{(m-2)}{2}}(x) h_{\alpha \beta}(u) \frac{\partial u^{\alpha}}{\partial x_{i}} \frac{\partial u^{\beta}}{\partial x_{i}} \mathrm{~d} x .
$$

The conditions that we will assume for $f$ are as follows:

$\left(f_{1}\right) f \in C^{1}\left(R^{m}\right), f>0$ on $R^{m}$.

$\left(f_{2}\right)$

$$
\frac{\partial f}{\partial x_{i}} x_{i} \geqq-(m-2) f \text { on } R^{m} .
$$

$\left(f_{3}\right)$ there are constants $\sigma>0, R_{0}>0$, such that

$$
\frac{(m-2)}{2} \frac{\partial f}{\partial x_{i}} x_{i} \geqq(\sigma-(m-2)) f \text { for }|x| \geqq R_{0} .
$$

$\left(f_{4}\right)$ for the constants $\sigma, R_{0}$ in $\left(f_{3}\right)$, there is a constant $C>0$ such that

$$
f^{\frac{(m-2)}{2}}(x) \leqq C|x|^{\sigma-(m-2)} \text { for }|x|>R_{0} .
$$

$\left(f_{5}\right)$ there are constants $C>0, R_{0}>0$, such that

$$
f^{\frac{(m-2)}{2}}(x) \leqq C|x|^{-(m-2)} \log |x| \text { for }|x|>R_{0} .
$$

For a map $u: R^{m} \rightarrow\left(N^{n}, h\right)$, an open set $U \subset N^{n}$, by $u(x) \in U$ as $|x| \rightarrow \infty$, we mean that there is $R>0$, such that $u\left(R^{m} \backslash B(R)\right) \subset U$. Also by $u \rightarrow p_{0} \in N^{n}$ as $|x| \rightarrow \infty$, we mean $u(x) \in U$ as $|x| \rightarrow \infty$ for any open set $U$ containing $p$.

We will prove a theorem which is slightly more general than Theorem A.

Theorem 1 Let $u:\left(R^{m}, f g_{0}\right) \rightarrow\left(N^{n}, h\right)$ be a $C^{2}$ harmonic map, $m>2$. $f$ satisfies $\left(f_{1}\right)-\left(f_{4}\right)$. If $u(x) \rightarrow p_{0} \in N^{n}$ as $|x| \rightarrow \infty$, then $u$ must be a constant map.

Remark $I$ It is easy to see that Theorem A is the special case of Theorem 1 when $f \equiv 1$ on $R^{m}$.

Theorem 2 Suppose $f$ satisfies $\left(f_{1}\right),\left(f_{2}\right)$ and $\left(f_{5}\right)$. Then for any $p \in N^{n}$, there is an (nonempty) open neighbourhood $U_{p} \subset N^{n}$, such that the family of open sets $\left\{U_{p} \mid p \in N^{n}\right\}$ has following property:

If $u:\left(R^{m}, f g_{0}\right) \rightarrow\left(N^{n}, h\right)$ is a $C^{2}$ harmonic map, $m>2$, and for some $p \in N^{n}$, $u(x) \in U_{p}$ as $|x| \rightarrow \infty$, then $u$ is a constant map.

Theorem 3 Suppose the sectional curvature of $N^{n}$ is bounded from above. Then for any $p \in N^{n}$, there is an (nonempty) open neighbourhood $U_{p} \subset N^{n}$, such that the family of open sets $\left\{U_{p} \mid p \in N^{n}\right\}$ has following property: 
If $u:\left(R^{m}, g_{0}\right) \rightarrow\left(N^{n}, h\right)$ is a $C^{2}$ harmonic map, $m>2$, and for some $p \in N^{n}$, $u(x) \in U_{p}$ as $|x| \rightarrow \infty$, then $u$ is a constant map.

\section{Proofs}

Before we start the proofs, we quote two results that we will need. The first result is about the finiteness of the energy.

Lemmas [11, Corollary 1] Let $u:\left(R^{m}, f_{0}\right) \rightarrow\left(N^{n}, h\right)$ be a $C^{2}$ harmonic map, $m>2$. $f$ satisfies $\left(f_{1}\right),\left(f_{2}\right)$. If $u$ is not a constant map, then the energy $E(u)$ must be infinite.

Proposition [9, Theorem 2.2] Let $u:\left(R^{m}, g_{0}\right) \rightarrow\left(N^{n}, h\right)$ be a $C^{2}$ harmonic map, $m>2$. Then there is a constant $\varepsilon_{0}>0$ depending only on $m$ and the upper bound of the sectional curvature of $N^{n}$ such that: if

$$
R^{2-m} \int_{B(R)} e(u) \leqq \varepsilon_{0}
$$

we have the inequality

$$
\sup _{B(R / 2)} e(u) \leqq C R^{-m} \int_{B(R)} e(u)
$$

where $C$ is a constant only depending on $m$ and the upper bound of the sectional curvature of $N^{n}$.

Proof of Theorem 1 We will prove the conclusion by contradiction.

Set

$$
E_{R}(u)=\int_{B(R)} e(u) \mathrm{dvol}_{M^{m}} .
$$

Suppose the harmonic map is not a constant map. Then by the lemma the energy of $u$ must be infinite. That is $E_{R}(u) \rightarrow \infty$, as $R \rightarrow \infty$.

We first derive an upper bound for the growth rate of $E_{R}(u)$ as $R \rightarrow \infty$.

Choose a local coordinate neighbourhood $U$ of $p_{0}$ in $N^{n}$, such that $p_{0}=0$, it is clear that we can choose the $U$ in such a way that

$$
h=h_{\alpha \beta}(y) \mathrm{d} y^{\alpha} \otimes \mathrm{d} y^{\beta}, \quad y \in U,
$$

satisfies (for two $n \times n$ matrices $A, B$, by $A \geqq B$, we mean that $A=B+D$ for a positive semi-definite matrix $D$ )

$$
\left(\frac{\partial h_{\alpha \beta}(y)}{\partial y^{\gamma}} y^{\gamma}+2 h_{\alpha \beta}(y)\right) \geqq\left(h_{\alpha \beta}(y)\right) \text { on } U \text {. }
$$

Now since $u(x) \rightarrow 0$ as $|x| \rightarrow \infty$, there is a $R_{1}$ such that for $|x|>R_{1}, u(x) \in U$, and

$$
\left(\frac{\partial h_{\alpha \beta}(u)}{\partial y^{\gamma}} u^{\gamma}+2 h_{\alpha \beta}(u)\right) \geqq\left(h_{\alpha \beta}(u)\right) \text { for }|x|>R_{1} \text {. }
$$

For $w \in C_{0}^{2}\left(R^{m} \backslash B\left(R_{1}\right), R^{n}\right)$, by the definition of harmonic maps we have

$$
\left.\frac{\mathrm{d}}{\mathrm{d} t}\right|_{t=0} E(u+t w)=0 \text {. }
$$


Then from (2), we have

$$
\int_{R^{m} \backslash B\left(R_{1}\right)}\left(2 h_{\alpha \beta}(u) \frac{\partial u^{\alpha}}{\partial x_{i}} \frac{\partial w^{\beta}}{\partial x_{i}}+\frac{\partial h_{\alpha \beta}(u)}{\partial y^{\gamma}} w^{\gamma} \frac{\partial u^{\alpha}}{\partial x_{i}} \frac{\partial u^{\beta}}{\partial x_{i}}\right) f^{\frac{(m-2)}{2}}(x) \mathrm{d} x=0 .
$$

Choose $w(x)=\phi(|x|) u(x)$ in (4) for $\phi(t) \in C_{0}^{\infty}\left(R_{1}, \infty\right)$, we have

$$
\begin{gathered}
\int_{R^{m} \backslash B\left(R_{1}\right)}\left(2 h_{\alpha \beta}(u)+\frac{\partial h_{\alpha \beta}(u)}{\partial y^{\gamma}} u^{\gamma}\right) \frac{\partial u^{\alpha}}{\partial x_{i}} \frac{\partial u^{\beta}}{\partial x_{i}} \phi(|x|) f^{\frac{(m-2)}{2}}(x) \mathrm{d} x \\
=-\int_{R^{m} \backslash B\left(R_{1}\right)} 2 h_{\alpha \beta}(u) \frac{\partial u^{\alpha}}{\partial x_{i}} u^{\beta} \frac{\partial \phi(|x|)}{\partial x_{i}} f^{\frac{(m-2)}{2}}(x) \mathrm{d} x .
\end{gathered}
$$

It is clear that (5) holds for Lipschitz function $\phi$ with compact support.

For $0<\varepsilon \leqq 1$, define

$$
\varphi_{\varepsilon}(t)= \begin{cases}1 & t \leqq 1 ; \\ 1+\frac{1-t}{\varepsilon} & 1<t<1+\varepsilon ; \\ 0 & t \geqq 1+\varepsilon .\end{cases}
$$

In (5), choose

$$
\phi(|x|)=\varphi_{\varepsilon}\left(\frac{|x|}{R}\right)\left(1-\varphi_{1}\left(\frac{|x|}{R_{1}}\right)\right), \quad R>2 R_{1} .
$$

Let $\varepsilon \rightarrow 0$, notice

$$
\frac{\partial \varphi_{\varepsilon}\left(\frac{|x|}{R}\right)}{\partial x_{i}}=-\frac{1}{R \varepsilon} \frac{x_{i}}{|x|} \text { for } R<|x|<R(1+\varepsilon),
$$

we get $\left(R_{2}=2 R_{1}, v=\left(v^{1}, \ldots, v^{m}\right)\right.$ is the outer normal on $\partial B(R)$.)

$$
\begin{gathered}
\int_{B(R) \backslash B\left(R_{2}\right)}\left(2 h_{\alpha \beta}(u)+\frac{\partial h_{\alpha \beta}(u)}{\partial y^{\gamma}} u^{\gamma}\right) \frac{\partial u^{\alpha}}{\partial x_{i}} \frac{\partial u^{\beta}}{\partial x_{i}} f^{\frac{(m-2)}{2}}(x) \mathrm{d} x+D\left(R_{1}\right) \\
=\int_{\partial B(R)} 2 h_{\alpha \beta}(u) \frac{\partial u^{\alpha}}{\partial x_{i}} v^{i} u^{\beta} f^{\frac{(m-2)}{2}}(x) \mathrm{d} S
\end{gathered}
$$

where

$$
\begin{aligned}
D\left(R_{1}\right)= & \int_{B\left(R_{2} \backslash B\left(R_{1}\right)\right.}\left(2 h_{\alpha \beta}(u)+\frac{\partial h_{\alpha \beta}(u)}{\partial y^{\gamma}} u^{\gamma}\right) \frac{\partial u^{\alpha}}{\partial x_{i}} \frac{\partial u^{\beta}}{\partial x_{i}}\left(1-\varphi_{1}\left(\frac{|x|}{R_{1}}\right)\right) f^{\frac{(m-2)}{2}}(x) \mathrm{d} x \\
& -\int_{B\left(R_{2}\right) \backslash B\left(R_{1}\right)} 2 h_{\alpha \beta}(u) \frac{\partial u^{\alpha}}{\partial x_{i}} u^{\beta} \frac{\partial \varphi_{1}\left(\frac{|x|}{R_{1}}\right)}{\partial x_{i}} f^{\frac{(m-2)}{2}}(x) \mathrm{d} x .
\end{aligned}
$$

Set

$$
Z(R)=\int_{B(R) \backslash B\left(R_{2}\right)} h_{\alpha \beta}(u) \frac{\partial u^{\alpha}}{\partial x_{i}} \frac{\partial u^{\beta}}{\partial x_{i}} f^{\frac{(m-2)}{2}}(x) \mathrm{d} x+D\left(R_{2}\right) \text { for } \quad R>R_{2} .
$$


Then

$$
Z^{\prime}(R)=\int_{\partial B(R)} h_{\alpha \beta}(u) \frac{\partial u^{\alpha}}{\partial x_{i}} \frac{\partial u^{\beta}}{\partial x_{i}} f^{\frac{(m-2)}{2}}(x) \mathrm{d} S .
$$

Also by Schwarz inequality, we have

$$
\begin{gathered}
\int_{\partial B\{R\}} h_{\alpha \beta}(u) \frac{\partial u^{\alpha}}{\partial x_{i}} v^{i} u^{\beta} f^{\frac{(m-2)}{2}}(x) \mathrm{d} S \\
\leqq C \sqrt{\left(\int_{\partial B(R)} h_{\alpha \beta}(u) \frac{\partial u^{\alpha}}{\partial x_{i}} \frac{\partial u^{\beta}}{\partial x_{i}} f^{\frac{(m-2)}{2}}(x) \mathrm{d} S\right)} \\
\times \sqrt{\left(\int_{\partial B(R)} h_{\alpha \beta}(u) u^{\alpha} u^{\beta} f^{\frac{(m-2)}{2}}(x) \mathrm{d} s\right)} .
\end{gathered}
$$

Since $E(u) \rightarrow \infty$ as $R \rightarrow \infty$, there is $R_{3} \geqq R_{2}$, such that $Z(R)>0$ for $R \geqq R_{3}$. Then (3), (7) and (8) imply

$$
Z(R)^{2} \leqq C Z^{\prime}(R)\left(\int_{\partial B(R)} h_{\alpha \beta}(u) u^{\alpha} u^{\beta} f^{\frac{(m-2)}{2}}(x) \mathrm{d} S\right) \text { for } \quad R>R_{3} .
$$

Denote

$$
M(R)=\int_{\partial B(R)} h_{\alpha \beta}(u) u^{\alpha} u^{\beta} f^{\frac{(m-2)}{2}}(x) \mathrm{d} S .
$$

Then for $R_{4} \geqq R \geqq R_{3}$,

$$
\int_{R}^{R_{4}}\left(\frac{-1}{Z(r)}\right)^{\prime} \mathrm{d} r \geqq C \int_{R}^{R_{4}} \frac{1}{M(r)} \mathrm{d} r .
$$

Let $R_{4} \rightarrow \infty$, notice $Z(R)>0$, we have

$$
\frac{1}{Z(R)} \geqq C \int_{R}^{\infty} \frac{1}{M(r)} \mathrm{d} r
$$

i.e.

$$
Z(R) \leqq C \frac{1}{\int_{R}^{\infty} \frac{1}{M(r)} \mathrm{d} r} \text { for } R>R_{3}
$$

By $\left(f_{4}\right)$ and the fact that $u(x) \rightarrow 0$ as $|x| \rightarrow \infty$, we have

$$
M(R) \leqq C \int_{\partial B(R)} h_{\alpha \beta}(u) u^{\alpha} u^{\beta} R^{\sigma-(m-2)} \mathrm{d} S \leqq C \eta(R) R^{\sigma+1}
$$

where $\eta(R)$ is chosen in such a way that

(i) $\eta(R)$ is nonincreasing on $\left(R_{3}, \infty\right)$;

(ii) $\eta(R) \rightarrow 0$ as $R \rightarrow \infty$;

(iii)

$$
\eta(R) \geqq \max _{|x|=R}\left\{h_{\alpha \beta}(u) u^{\alpha} u^{\beta}\right\}
$$


Then

$$
\int_{R}^{\infty} \frac{1}{M(r)} \mathrm{d} r \geqq \frac{C}{\eta(R)} \int_{R}^{\infty} \frac{1}{r^{1+\sigma}} \mathrm{d} r=\frac{C}{\eta(R)} R^{-\sigma}
$$

Thus

$$
Z(R) \leqq C \eta(R) R^{\sigma} \quad \text { for } \quad R \geqq R_{3}
$$

Therefore

$$
E_{R}(u) \leqq C\left(\eta(R)+\frac{c(u)}{R^{\sigma}}\right) R^{\sigma}
$$

where $c(u)$ is a constant depending on the harmonic map $u$. This gives an upper bound for the growth rate of $E_{R}(u)$. In the following we will derive a lower bound for the growth rate of $E_{R}(u)$.

In fact, if $\left(f_{3}\right)$ holds for $R_{0}=0$, by the proof of Theorem 10 in [4], we have that if the map $u$ is not a constant map, then for $R$ large enough,

$$
E_{R}(u) \geqq c_{1}(u) R^{\sigma} \quad \text { for some constant } c_{1}(u)>0 .
$$

Now a contradiction appears as $R \rightarrow \infty$ from (11) and (12). If we only assume $\left(f_{3}\right)$ holds for $R_{0}>0$, the proof in [4] still gets through by a little bit of modification. Here we only indicate the necessary changes in the proof of [4]:

Under the assumption $\left(f_{3}\right)$, if we proceed as in the proof of Theorem 10 in [4], we have

$$
R \int_{\delta B(R)} e(u) \mathrm{d} S+H\left(R_{0}\right)-\sigma \int_{B(R) \backslash B\left(R_{0}\right)} e(u) \mathrm{d} x \geqq 0 \text { for } \quad R>R_{0}
$$

where $H\left(R_{0}\right)$ only depends on the $R_{0}$ and $u$. Then for some function $H_{1}\left(R_{0}\right)$ and $R>R_{0}$,

$$
R\left\{E_{R}(u)+\frac{1}{\sigma} H_{1}\left(R_{0}\right)\right\}^{\prime}-\sigma\left\{E_{R}(u)+\frac{1}{\sigma} H_{1}\left(R_{0}\right)\right\} \geqq 0
$$

Thus

$$
\left\{R^{-\sigma}\left(E_{R}(u)+\frac{1}{\sigma} H_{1}\left(R_{0}\right)\right)\right\}^{\prime} \geqq 0 \quad \text { for } \quad R>R_{0} .
$$

Since $E_{R}(u) \rightarrow \infty$ as $R \rightarrow \infty$, there is $R_{5}>R_{0}$ such that

$$
c_{2}(u)=R_{5}^{-\sigma}\left(E_{R_{5}}(u)+\frac{1}{\sigma} H_{1}\left(R_{0}\right)\right)>0 .
$$

Then

$$
E_{R}(u)+\frac{1}{\sigma} H_{1}\left(R_{0}\right) \geqq c_{2}(u) R^{\sigma} \text { for } \quad R>R_{5}
$$

Now it is clear that (12) is true. $\square$ 
Proof of Theorem 2. The proof of Theorem 2 is from a modification of the proof of Theorem 1. Choose a family of coordinate neighbourhoods $\left\{U_{p} \mid p \in N^{n}\right\}$ as follows: for $p \in N^{n}$, choose a coordinate chart $U_{p}$, such that $p=0$,

$$
\left(\frac{\partial h_{\alpha \beta}(y)}{\partial y^{\gamma}} y^{\gamma}+2 h_{\alpha \beta}(y)\right) \geqq\left(h_{\alpha \beta}(y)\right) \text { on } \quad U_{p} ;
$$

and

$$
h_{\alpha \beta}(y) y^{\alpha} y^{\beta} \leqq C_{p}
$$

where $C_{p}$ is an arbitrary constant which may depend on $p$.

Then we claim that this family $\left\{U_{p} \mid p \in N^{n}\right\}$ is what we want.

In fact, if $u:\left(R^{m}, f g_{0}\right) \rightarrow\left(N^{n}, h\right)$ is a non-constant $C^{2}$ harmonic map, and for some $p \in N^{n}, u(x) \in U_{p}$ as $|x| \rightarrow \infty$, then we may assume that for some $R_{0}, u(x) \in U_{p}$ for $|x|>R_{0}$. We proceed as in the proof of Theorem 1, we get

$$
\frac{1}{Z(R)} \geqq C \int_{R}^{\infty} \frac{1}{M(r)} \mathrm{d} r \quad \text { for } \quad R>R_{3} .
$$

But in this case

$$
M(R) \leqq C \int_{\partial B(R)} h_{\alpha \beta}(u) \mathcal{u}^{\alpha} u^{\beta} R^{-(m-2)} \log R \mathrm{~d} S \leqq C C_{p} R \log R .
$$

Therefore

$$
\int_{R}^{\infty} \frac{1}{M(r)} \mathrm{d} r \geqq \frac{1}{C C_{p}} \int_{R}^{\infty} \frac{1}{r \log r} \mathrm{~d} r=\infty .
$$

Now we have a contradiction to (13), since if $u$ is not a constant map, $Z(R)>0$ for $R$ large.

Remark 2 In the proof of (9) and (13), it is crucial to use the fact that $Z(R)>0$ for $R$ large, which is guaranteed by the lemma. Otherwise one might get false information. For example, take a harmonic map $u: S^{m} \rightarrow N^{n}$, there is a positive function $f$, such that $\left(R^{m}, f g_{0}\right)=S^{m} \backslash\{p\}$, then we have a map $u:\left(R^{m}, f g_{0}\right) \rightarrow N^{n}$. But we can not follow the proof of Theorem 2 to conclude that $u$ is a constant map, because it is no longer true that $Z(R)>0$ for $R$ large.

Remark 3 The proof of Theorem 2 is similar to ideas used in [8].

Proof of Theorem 3 For any $p \in N^{n}$, we choose a coordinate neighbourhood $U_{p}$ of $p$ in such a way that $p=0$,

$$
\left(\frac{\partial h_{\alpha \beta}(y)}{\partial y^{\gamma}} y^{\gamma}+2 h_{\alpha \beta}(y)\right) \geqq\left(h_{\alpha \beta}(y)\right) \text { on } \quad U_{p}
$$

and

$$
h_{\alpha \beta}(y) y^{\alpha} y^{\beta} \leqq C_{1} \quad \text { on } \quad U_{p}
$$

where $C_{1}$ is a constant not depending on $u$ and will be specified in a moment.

Now we say that this family of coordinate neighbourhoods $\left\{U_{p} \mid p \in N^{n}\right\}$ is what we want. 
In fact, if $u:\left(R^{m}, g_{0}\right) \rightarrow\left(N^{n}, h\right)$ is a non-constant $C^{2}$ harmonic map, and for some $p \in N^{n}, u(x) \in U_{p}$ as $|x| \rightarrow \infty$, then we may assume that for some $R_{0}, u(x) \in U_{p}$ for $|x|>R_{0}$. Now we proceed as in the proof of Theorem 1 for the special case that $f \equiv 1, \sigma=m-2$, we get

$$
E_{R}(u) \leqq C\left(\sup _{R \leqq t<\infty}\left\{\max _{|x|=t}\left\{h_{\alpha \beta}(u) u^{\alpha} u^{\beta}\right\}\right\}+\frac{c(u)}{R^{m-2}}\right) R^{m-2} .
$$

If we choose $C_{1}$ in (15) in such a way that

$$
C_{1} C \leqq \frac{\varepsilon_{0}}{2}
$$

where $\varepsilon_{0}$ is defined in the proposition. Then we can choose $R_{6}$ large enough, so that

$$
C\left(\sup _{R \leqq t<\infty}\left\{\max _{|x|=t}\left\{h_{\alpha \beta}(u) u^{\alpha} u^{\beta}\right\}\right\}+\frac{c(u)}{R^{m-2}}\right) \leqq \varepsilon_{0} \quad \text { for } \quad R>R_{6},
$$

i.e.

$$
R^{2-m} E_{R}(u) \leqq \varepsilon_{0} \quad \text { for } \quad R>R_{6} .
$$

Now the proposition implies

$$
\sup _{B(R / 2)} e(u) \leqq C R^{-m} \int_{B(R)} e(u) \text { for } R>R_{6}
$$

i.e.

$$
C R^{m} \sup _{B(R / 2)} e(u) \leqq E_{R}(u) \text { for } R>R_{6}
$$

This is clearly a contradiction to (16) if the harmonic map $u$ is not a constant map.

Acknowledgement. The author would like to thank Professor Jerry Kazdan for helpful suggestions.

Added in the proofs. After this research was completed, the author learned from Dong Zhang that by using an argument due to Giaquinta and Hildebrandt (see pp. 334-335 in [9]), one can prove that the open sets in the Theorem B can be chosen as the "small geodesic balls" $B_{p}(r)$ with $r<\frac{\pi}{2} \sqrt{C_{N}}$, where $C_{N}$ is an upper bound of the sectional curvature of $N^{n}$. For details on this point, see a coming paper by Dong Zhang.

\section{References}

1. Cheng, S.Y.: Liouville theorem for harmonic maps. (Proc. Symp. Pure Math. Uni. Hawaii, Honolulu, Hawaii, 1979). In: Proc. Symp. Pure Math., XXXVI, pp. 147-151. Providence, R.I.: Am. Math. Soc. 1980

2. Eells, J., Lemaire, L.: Another report on harmonic maps. Bull. Lond. Math. Soc. 20, 385-524 (1988)

3. Garber, W.D., Ruijsenaars, S.N.M., Seiler, E., Burns, D.: On finite action solutions of the nonlinear $\sigma$-model. Ann. Phys. 119, 305-325 (1979)

4. Hildebrandt, S.: Liouville theorems for harmonic mappings, and an approach to Beinstein theorems. Ann. Math. Stud. 102, 107-131 (1982) 
5. Hildebrandt, S., Jost, J., Widman, K.O.: Harmonic mappings and minimal submanifolds. Invent. Math. 62, 269-298 (1980)

6. Hildebrandt, S., Kaul, H.: Two dimensional variational problems with obstructions, and Plateau's problem for $H$-surfaces in a Riemannian manifold. Commun. Pure Appl. Math. 25, 187-223 (1972)

7. Hildebrandt, S., Kaul, H., Widman, K.O.: An existence theorem for harmonic mappings of Riemannian manifolds. Acta Math. 138, 1-16 (1977)

8. Hwang, J.F.: Comparison principles and Liouville theorems for prescribed mean curvature equations in unbounded domain. Ann. Sc. Norm. Super. Pisa XI3, 341-355 (1988)

9. Schoen, R.: Analytic aspects of the harmonic map problem. In: Chen, S.S., (ed.) Seminar on nonlinear partial differential equations. pp. 321-358, 1984

10. Schoen, R., Yau, S.T.: Harmonic maps and the topology of stable hypersurfaces and manifolds with non-negative Ricci curvature. Comm. Math. Helv. 51, 333-341 (1976)

11. Sealey, H.C.J.: Some conditions ensuring the vanishing of harmonic differential forms with applications to harmonic maps and Yang-Mills theory. Math. Proc. Camb. Philos. Soc. 91, 441-452 (1982)

12. Yau, S.T.: Harmonic functions on complete Riemannian manifolds. Commun. Pure Appl. Math. 28, 201-228 (1975) 